\title{
Percepción de equidad de género y equilibrio trabajo-familia en trabajadores pertenecientes a empresas públicas y privadas de Chile
}

\section{Perception of gender equity and work-family balance in workers belonging to public and private companies in Chile}

\section{Percepção da igualdade de gênero e do equilíbrio entre trabalho e família em trabalhadores de empresas públicas e privadas no Chile}

\author{
Andrés Jiménez Figueroa ${ }^{1}$, ORCID 0000-0001-8324-0250 \\ Agustina Hernández Reveco ${ }^{2}$, ORCID 0000-0003-2249-3101 \\ ${ }^{12}$ Facultad de Psicología, Universidad de Talca. Chile
}

Resumen: Se analiza si existen diferencias significativas en la Percepción de Equidad de Género y Equilibrio Trabajo-Familia en trabajadores pertenecientes a empresas públicas y privadas. A 300 trabajadores de empresas públicas y privadas, se les aplicaron los instrumentos, Equilibrio TrabajoFamilia (Moreno, Sanz, Rodríguez \& Geurts, 2009) y Percepción de Equidad de Género (Gómez \& Jiménez, 2015). Se observa que existen diferencias estadísticamente significativas entre los trabajadores pertenecientes a empresas públicas y privadas en los niveles de Percepción de Equidad de Género y Equilibrio Trabajo-Familia, donde los trabajadores de empresas privadas tienen una mayor Percepción de Equidad de Género. Asimismo, presentan una mayor percepción de Equilibrio Trabajo-Familia. Se presentan correlaciones significativas y positivas entre Percepción de Equidad de Género y Equilibrio Trabajo-Familia. Se concluye que las empresas presentan diferencias en sus accionar en pro de establecer las buenas prácticas de conciliación que repercuten en la percepción de equidad.

Palabras clave: percepción de equidad de género, equilibrio trabajo-familia, empresas públicas, empresas privadas

Abstract: It is analyzed whether there are significant differences in the Perception of Gender Equity and Work-Family Balance in workers belonging to public and private companies. The instruments Work-Family Balance (Moreno, Sanz, Rodríguez \& Geurts, 2009) and Gender Equity Perception (Gómez \& Jiménez, 2015) were applied to 300 workers from public and private companies. It is observed that there are statistically significant differences between workers belonging to public and private companies in the levels of Gender Equity Perception and Work-Family Balance, where workers in private companies have a higher Gender Equity Perception. Likewise, they have a higher perception of Work-Family Balance. There are significant and positive correlations between the perception of gender equity and work-family balance. It is concluded that the companies present differences in their actions in favor of establishing good reconciliation practices that have an impact on the perception of equity.

Keywords: gender equity perception, work-family balance, public companies, private companies 
Resumo: Se analisa a existência de diferenças significativas na percepção de equidade de gênero e equilíbrio entre trabalho e família em trabalhadores de empresas públicas e privadas. Para 300 trabalhadores de empresas públicas e privadas, foram aplicados os instrumentos de Equilíbrio TrabalhoFamília (Moreno, Sanz, Rodríguez \& Geurts, 2009) e Percepção da Equidade de Gênero (Gómez \& Jiménez, 2015). Observa-se que existem diferenças estatisticamente significativas entre trabalhadores de empresas públicas e privadas nos níveis de Percepção da Equidade de Gênero e Equilíbrio TrabalhoFamília, na qual trabalhadores de empresas privadas apresentam maior Percepção de Equidade de Gênero, assim como, apresentam uma maior percepção do Equilíbrio Trabalho-Família. São apresentadas correlações significativas e positivas entre a Percepção da Equidade de Gênero e o Equilíbrio entre Trabalho e Família. Conclui-se que as empresas apresentam diferenças em suas ações em pro de estabelecer boas práticas de conciliação que impactam a percepção da equidade.

Palavras-chave: percepção de eqüidade de gênero, equilíbrio trabalho-família, empresas públicas, empresas privadas

Cómo citar:

Jiménez Figueroa, A., \& Hernández Reveco, A. (2020). Percepción de equidad de género y equilibrio trabajo-familia en trabajadores pertenecientes a empresas públicas y privadas de Chile. Ciencias Psicológicas, 14(2), e-2201. doi: https://doi.org/10.22235/cp.v14i2.2201

Correspondencia: Andrés Jiménez Figueroa, Facultad de Psicología, Universidad de Talca. Chile. Avda. Lircay s/n, Talca, Chile. E-Mail: anjimenez@utalca.cl. Agustina Hernández Reveco, Universidad de Talca. E-Mail: agus.hndez.r@gmail.com

\section{Introducción}

El trabajo y la familia son dos ámbitos importantes para el desarrollo personal y social de los ciudadanos (Marín, Infante \& Rivero, 2002). Una de las problemáticas más grandes y comunes dentro del mundo del trabajo ha sido el acceso de la mujer al mundo laboral. Este proceso ha provocado cambios en la historia del rol que ha ejercido la mujer dentro de la sociedad, el cual está relacionado con labores domésticas y de crianza, mientras que el del hombre tiene relación con el rol proveedor y sostenedor de la familia. Esta concepción ha ido variando en los últimos años, debido a cambios en la estructura familiar y por el mayor acceso de la mujer a la educación y flexibilización en las condiciones de trabajo (Jiménez \& Moyano, 2008).

Según Gómez y Martí (2004) con el ingreso de la mujer al trabajo remunerado se han presentado dificultades para las familias, dado que tanto el hombre como la mujer deben dedicar tiempo proporcionalmente mayor al trabajo que al hogar, instalándose el cansancio y estrés. Equilibrar el trabajo y la familia con la obligación de admitir la variedad de roles crean conflicto y estrés en la persona (Riquelme, Rojas \& Jiménez, 2012).

En relación a la redistribución de roles dentro de la familia, que surge a partir de la incorporación de la mujer al ámbito laboral, es importante mencionar la responsabilidad que tienen las organizaciones en cuanto a la conciliación trabajo-familia por medio de la implementación de prácticas organizacionales encargadas específicamente en estas áreas (Caballer, Peiró \& Sora, 2011). Estas políticas implementadas por las organizaciones favorecen la conciliación trabajo-familia percibida por los trabajadores, quienes a su vez mejoran el 
rendimiento en sus labores (Anderson, Coffey \& Byerly, 2002). Las apreciaciones que tengan los trabajadores acerca del apoyo otorgado por su organización en los temas de familia, se relaciona con la percepción de apoyo organizacional (Allen, 2001). Así lo muestra un estudio realizado por Besarez, Jiménez y Riquelme (2014) con 137 trabajadores que compara empresas con y sin políticas organizacionales en temas de equilibrio trabajo-familia, en el cual se observó que sí había diferencias significativas en cuanto a el apoyo organizacional percibido hacia la familia y la satisfacción laboral.

Es por esto que las empresas implementan políticas organizacionales que asumen la responsabilidad social, resguardando la calidad de vida de los trabajadores. Estas políticas son señaladas, según Servicio Nacional de la Mujer [SERNAM] (2003) como todas aquellas iniciativas, adicionales a las que establece la ley, adoptadas por las organizaciones con el objetivo de contribuir a las buenas prácticas laborales y así, mejorar el rendimiento y adhesión de los empleados con la empresa (Abarca \& Errázuriz, 2007). Con el mismo fin se crean las prácticas de equidad de género o conciliación trabajo y familia. Una de las iniciativas para enriquecer la equidad de género dentro de las empresas fue implementada por SERNAM (2017) al crear entre los años 2007 y 2012 el modelo IGUALA, el cual sigue un plan que es transversal para empresas tanto públicas como privadas, como también para grandes, pequeñas y medianas empresas (PYMES), contemplando a todos los trabajadores pertenecientes a la organización y enfocado en la erradicación de las diferencias de género. Entre las acciones que se llevaron a cabo, por medio de este modelo, se destacan (SERNAM, 2017, p. 8):

El compromiso para el aumento de dotación femenina y la igualdad de oportunidades entre mujeres y hombres en el proceso de selección y de promoción interna. El empeño para lograr equipos mixtos equilibrados, asegurando cupos para las mujeres en las actividades de capacitación comúnmente dirigidas a hombres. La realización de actividades informativas y formativas sobre la importancia de la igualdad en las relaciones laborales y la prevención de la violencia y el acoso sexual y laboral. El desarrollo de iniciativas para la protección de la maternidad, es decir, por ejemplo, la organización de los horarios de trabajo para las mujeres embarazadas. La implementación de acciones de conciliación de la vida laboral, familiar y personal a través de un retorno progresivo del postnatal hasta los 9 meses de la/el hija/o. La ampliación del postnatal masculino sumándole tres días adicionales a los que considera la ley. La habilitación de servicios de guardería y tutoría escolar para hijas/os de trabajadoras/es; y, la implementación de una jornada de trabajo flexible.

El modelo tuvo una aceptación del $79 \%$ de los trabajadores chilenos encuestados en el año 2011, quienes presentan cambios positivos ante el enfoque de equidad de género. Por otro lado, en Chile, existe una plataforma nacional, denominada Iniciativa de Paridad de Género (IPG), que es llevada a cabo por parte de las autoridades pertenecientes al gobierno y representantes del sector privado. Con el objetivo de ingresar a más mujeres a la economía y dar término a las desigualdades de género, tanto en el sector público como en el privado (Comunidad Mujer, 2016). Es importante señalar que, integrar la mirada de la perspectiva de género en las empresas, genera el entusiasmo por crear prácticas orientadas al desarrollo femenino, en donde se puedan sentir en igualdad de condiciones, afianzando su compromiso con la organización (Stevens \& Van Lamoen, 2001).

No implementar medidas laborales encargadas específicamente en temas de conciliación familiar y laboral, perjudica la calidad de vida del trabajador, reflejándose en deseos de abandono, cuando éste se ve interferido por la incompatibilidad de las labores familiares (Boles, Johnston \& Hair, 1997) y la disminución de la satisfacción que le provoca su empleo, afectando su bienestar, el cual se refleja en problemas físicos, como la fatiga y estrés, y mentales, como la preocupación y culpabilidad (Greenglass, 1985).

La fuerte inserción de la mujer al mundo laboral no solo planteó problemas en las 
dinámicas familiares, sino también dentro de sus trabajos, en donde se presentan grandes diferencias de género. Así lo señala Comunidad Mujer (2016) cuando señala que la participación de la mujer en el mundo laboral no asegura igualdad con el género opuesto, ya que en este contexto se presentan varias discriminaciones, situaciones de segmentación y varias otras dificultades que van en desmedro hacia sus salarios (Instituto Nacional de Estadísticas, 2015) y en las oportunidades que éstas tienen para postular a cargos más importantes o de liderazgo. Bajo este punto se han implementado medidas que apuntan a las buenas prácticas laborales fomentando la equidad de género dentro de las organizaciones, puesto que, impulsar la igualdad entre hombres y mujeres promueve la eficiencia económica e incide positivamente sobre otros ámbitos del desarrollo económico o personal (Banco Mundial, 2012). Además, adoptar prácticas organizacionales que no sean equitativas entre el hombre y la mujer, puede provocar sentimientos de injusticia, los cuales afectan al rendimiento dentro de la empresa, provocando una desvinculación de sus labores (Lambert, Hogan \& Barton, 2001).

Por otro lado, el trabajo doméstico ha llegado a ser reconocido por organizaciones internacionales como un trabajo, el cual está muy relacionado con el concepto de doble jornada (Ruvalcaba, 2001). Esto se explica puesto que, el tiempo que se emplea en el trabajo se hace en virtud del tiempo de trabajo doméstico, creando así la doble jornada, y a medida que las mujeres vayan en aumento como trabajadoras asalariadas o no, lo harán en virtud de esta condición (Hirata \& Zariffian, 2007). Por su parte, la Organización para la Cooperación y Desarrollo Económicos [OCDE (2010)] reporta que en Chile presenta una alta cantidad de horas en que las personas pasan en su lugar de trabajo, siendo un total de 2000 horas al año aproximadamente. En cuanto a esto, el Instituto Nacional de Estadísticas (2016) evidencia que durante el año 2015 en Chile un $28.2 \%$ de las mujeres trabajaba en promedio de 1 a 30 horas semanales, mientras que las que trabajaban de 31 a 44 horas representaba el $19.9 \%$.

Al respecto, esta fuerte incorporación de la mujer al ámbito laboral brinda una nueva visión de la dinámica familiar, modificando los roles y añadiendo el rol de productora, si se observa desde la perspectiva de modelo de familia tradicional, se propone un nuevo concepto de familia. Se ha podido percibir, durante el último tiempo, los cambios que se han generado en las estructuras y dinámicas familiares. Factores tales como el mayor acceso a la educación, disminución de las tasas de fecundidad y la creciente necesidad de incorporación de ingresos económicos al hogar (Abramo \& Valenzuela, 2006) han modificado las formas de trabajo y empleo y ha impulsado la redistribución de los roles asignados al hombre y la mujer, sustituyendo el rol del varón como único proveedor del hogar, por la incorporación de la mujer al trabajo remunerado. Este cambio genera transformaciones en la vida económica, social y política, viéndose reflejada mayoritariamente en la distribución de responsabilidades entre el hombre y la mujer dentro del ámbito familiar, la cual genera conflictos entre el trabajo y la familia, por las exigencias e incompatibilidad que estos dos contextos provocan, ocasionando un desgaste en la energía de la persona (Guerrero, 2003). Además, es posible observar estas transformaciones en las personas que optan por vivir solas, en pareja y sin hijos, en hogares monoparentales y en uniones de mutuo acuerdo.

Cabe destacar que las desigualdades de género existentes corresponden al modelo patriarcal que dominaba a la sociedad chilena el cual, culturalmente hablando, diferencia los roles pertenecientes al hombre y la mujer, donde el primero centra su vida en el trabajo, siendo el sustento del hogar y reduciendo su participación en las labores familiares, y no así la mujer, la cual sigue el rol tradicional de emplear variados papeles, tales como dueña de casa, madre, esposa y trabajadora. Sin embargo, las distinciones existentes de género en la actualidad no solo vienen dictadas desde antes por concepto de sociedad, sino también por el prejuicio existente que hay acerca de la carencia de experiencia laboral (Heilman, 2001) y por la incapacidad de la mujer para desempeñarse de manera eficiente y constante en las labores de trabajo, puesto que éstas se llevan a cabo en edad fértil (Lorenzini et. al, 2013). 
Todo esto se ve reflejado por la creciente participación laboral masculina, que según la encuesta INE (2018) corresponde a un porcentaje de 71.1\%, en comparación a la de la mujer que es igual a un $49.3 \%$, por las diferencias de remuneraciones en el salario, la cual indica la encuesta ESI realizada el año 2017 que es igual a U\$228 mensuales entre hombres y mujeres (Durán \& Kremerman, 2018) y, por los distintos tipos de discriminaciones laborales, tales como la segregación horizontal, la cual hace referencia a los trabajos llevados a cabo por las mujeres que son escasos en actividad y profesión y, la segregación vertical, la cual se entiende como los empleos que se encuentran en la parte más baja de la jerarquía, según cargos dentro de una organización (Maruani, 1993). Una de las razones que explica esta problemática es la asignación del rol protector que se le entrega a la mujer ante el cuidado de los hijos, lo que las hace la principal responsable de los costos de la reproducción, ya que según Comunidad Mujer (2017):

La mayoría de las mujeres recibe salarios más bajos que los hombres debido al castigo que el empleador hace, al estar las mujeres en edad fértil, como anticipo del eventual costo que deberá enfrentar con el pre y post natal, el fuero maternal, el derecho de alimentación, la sala cuna y/o las ausencias en caso de enfermedades u otros, de los hijos/as pequeños (p. 10).

El objetivo de este trabajo es evaluar si existen diferencias en la percepción que tienen los trabajadores, pertenecientes a empresas públicas o privadas, acerca de las variables Equidad de Género y Equilibrio Trabajo-Familia dentro de sus empresas.

Los objetivos específicos son:

1. Determinar si existen diferencias en la percepción de Equidad de Género en los trabajadores de una empresa pública y otra privada.

2. Determinar si existen diferencias en relación al Equilibrio Trabajo-Familia en los trabajadores de una empresa pública y otra privada.

3. Describir la relación existente entre las variables Equidad de Género y Equilibrio Trabajo-Familia de la muestra total.

En base a los antecedentes reportados, se formularon las siguientes hipótesis:

H1: Existen diferencias estadísticamente significativas en la percepción de Equidad de Género entre una empresa pública y otra privada.

H2: Existen diferencias estadísticamente significativas en relación al Equilibrio TrabajoFamilia entre una empresa pública y otra privada.

H3: Existe una relación fuerte y positiva entre las variables Equidad de Género y Equilibrio Trabajo-Familia aplicadas a la muestra total.

\section{Método}

\section{Participantes}

El estudio contó con una muestra de 300 trabajadores, de los cuales 150 son mujeres y 150 hombres, distribuidos equitativamente entre una organización pública y otra privada. La muestra fue escogida de manera no probabilística y por conveniencia. Las edades de los particpantes fluctúan en un rango de 18 a 64 años con una media de 38 años. Del total de la muestra, el $35.7 \%$ está casado, el 33\% está soltero, el $21.7 \%$ dice estar en pareja, el $5.3 \%$ está separado, el $3 \%$ está divorciado, y finalmente un $0.7 \%$ es viudo. En cuanto a la cantidad de hijos que posee cada participante de la muestra, se puede apreciar que $29.7 \%$ no tiene hijos, el $55.3 \%$ tiene 1 o 2 hijos y el $11.3 \%$ tiene 3 o más hijos.

\section{Instrumentos}


Cuestionario SWING (Moreno, Sanz, Rodríguez \& Geurts, 2009). Este instrumento se compone de 22 ítems con una escala de tipo Likert con respuestas de 0 a 3, donde 0 es nunca, 1 a veces, 2 a menudo y 3 siempre. Los ítems se distribuyen en 4 sub-escalas, las cuales se presentan de la siguiente manera: Interacción negativa trabajo-familia (8 ítems), Interacción negativa familia-trabajo (4 ítems), Interacción positiva trabajo-familia (5 ítems), e Interacción positiva familia-trabajo (5 ítems). La validación española del instrumento realizada por Moreno et al. (2009) arrojó índices de Alfa de Cronbach entre .77 y .89, siendo estos indicadores de una alta fiabilidad del cuestionario.

Cuestionario de Percepción de Equidad de Género (Gómez \& Jiménez, 2015). Este cuestionario consta de 14 ítems tipo Likert, donde las respuestas van desde Muy de acuerdo a Muy en desacuerdo. Además, se distribuyen en 4 sub-escalas, las cuales son: Percepción de disponibilidad de tiempo personal (3 ítems), Percepción de distribución de responsabilidades de trabajo doméstico de cuidado (5 ítems), Percepción de distribución de responsabilidades de manutención económica del hogar (3 ítems), y Percepción de distribución de demandas laborales (3 ítems). La validación del instrumento realizada por Gómez y Jiménez (2015) arrojó un índice de Alfa de Cronbach de .87 siendo éste un indicador de alta fiabilidad del cuestionario (Jiménez \& Gómez, 2019).

\section{Procedimiento}

En primer lugar, se envió un correo institucional a empresas públicas y privadas, explicando el propósito de la investigación, a fin de obtener su apoyo y autorización. Luego, se realizó el contacto con las personas de la muestra, mediante reuniones con profesionales de Recursos Humanos, Subdirección, Relaciones Labores y jefa de Bienestar. Por último, se llevó a cabo la aplicación de la batería de instrumentos, invitando de forma personalizada y voluntaria a cada funcionario en su puesto de trabajo lo que se deja estipulado en un consentimiento informado, el cual se lee junto a los voluntarios antes de realizar el cuestionario, en formato papel.

\section{Diseño y análisis de datos}

Estudio correlacional de tipo transversal. Los datos fueron procesados y analizados mediante el programa estadístico SPSS, llevando a cabo análisis descriptivos y correlacionales. Debido a que se cumplió el supuesto de normalidad para la distribución de las variables en estudio (Kolmogorov Smirnov $p>$. 05), se utilizaron pruebas paramétricas.

\section{Resultados}

La tabla 1 muestra los estadísticos descriptivos para la variable Equidad de Género y Equilibrio Trabajo-Familia.

Tabla 1

Estadísticos descriptivos

\begin{tabular}{cccccc}
\hline Variable & $N$ & $M$ & $D E$ & Mín. & Máx. \\
\hline Equidad de Género & 300 & 54.96 & 14.07 & 23 & 103 \\
Equilibrio Trabajo-Familia & 300 & 40.21 & 15.59 & 4 & 84 \\
\hline
\end{tabular}


Con respecto a la variable Percepción de Equidad de Género, la empresa pública presenta una media de 43.14 y la empresa privada una media de 66.79 (ver Tabla 2). Por medio del análisis realizado mediante la prueba $T$ para muestras independientes en la que se ha asumido desigualdad de varianza $(F=20.75 ; p<.05)$ es posible señalar que existen diferencias estadísticamente significativas en relación a la Percepción de Equidad de Género entre la empresa pública y otra privada $(t=-26.90 ; p<.05)$ (ver Tabla 3$)$.

Tabla 2

Comparación de la Percepción de Equidad de Género de la muestra total

\begin{tabular}{lccccc}
\hline & Empresa & $N$ & $M$ & $D E$ & Error típ. de la $M$ \\
\cline { 2 - 6 } Equidad de Género & Pública & 150 & 43.14 & 5.70 & .46 \\
& & & & & \\
& Privada & 150 & 66.79 & 9.12 & .74 \\
\hline
\end{tabular}

Tabla 3

Diferencias de grupos para Percepción de Equidad de Género

\begin{tabular}{|c|c|c|c|c|c|c|c|c|c|}
\hline & \multicolumn{2}{|c|}{$\begin{array}{c}\text { Prueba de } \\
\text { Levene para la } \\
\text { igualdad de } \\
\text { varianzas }\end{array}$} & \multicolumn{7}{|c|}{ Prueba T para la igualdad de medias } \\
\hline & & & & & & & Error típ. & $\begin{array}{r}95 \% \text { Int } \\
\text { confian } \\
\text { dife }\end{array}$ & $\begin{array}{l}\text { rvalo de } \\
\text { a para la } \\
\text { ncia }\end{array}$ \\
\hline & $F$ & Sig. & $T$ & $g l$ & (bilateral) & de medias & diferencia & Superior & Inferior \\
\hline $\begin{array}{l}\text { Se han } \\
\text { asumido } \\
\text { varianzas } \\
\text { iguales }\end{array}$ & 20.75 & .000 & -26.90 & 298 & .000 & -23.64 & .87 & -25.37 & -21.91 \\
\hline $\begin{array}{c}\text { No se } \\
\text { han } \\
\text { asumido } \\
\text { varianzas } \\
\text { iguales }\end{array}$ & & & -26.90 & 249.9 & .000 & -23.64 & .87 & -25.37 & -21.91 \\
\hline
\end{tabular}


Con respecto a la variable Equilibrio Trabajo-Familia, la empresa pública presenta una media de 27.17 y la empresa privada una media de 53.25. Por medio del análisis realizado mediante la prueba $T$ para muestras independientes (ver Tabla 4) en la que se ha asumido desigualdad de varianza $(F=3.90 ; p<.05)$ es posible señalar que existen diferencias estadísticamente significativas en relación al Equilibrio Trabajo-Familia entre la empresa pública y otra privada $(t=-26.44 ; p<.05)$ (ver Tabla 5$)$.

Se puede apreciar que existe correlación fuerte y positiva entre las variables Equidad de género y Equilibrio Trabajo-Familia $(r=.796, p<.05)$.

Tabla 4

Comparación de la relación Equilibrio Trabajo-Familia de la muestra total

\begin{tabular}{lccccc}
\hline & Empresa & $N$ & $M$ & $D E$ & Error típ. de la $M$ \\
\hline \multirow{2}{*}{ Equilibrio Trabajo-Familia } & Pública & 150 & 27.17 & 7.59 & .61 \\
& Privada & 150 & 53.25 & 9.44 & .77 \\
\hline
\end{tabular}

Tabla 5

Diferencias de grupos para Equilibrio Trabajo-Familia

\begin{tabular}{|c|c|c|c|c|c|c|c|c|c|}
\hline & \multicolumn{2}{|c|}{$\begin{array}{c}\text { Prueba de } \\
\text { Levene para } \\
\text { la igualdad de } \\
\text { varianzas }\end{array}$} & \multicolumn{7}{|c|}{ Prueba $T$ para la igualdad de medias } \\
\hline & & & & & & & Error típ. & $\begin{array}{r}95 \% \text { Inte } \\
\text { confianz } \\
\text { difer }\end{array}$ & $\begin{array}{l}\text { valo de } \\
\text { para la } \\
\text { ncia }\end{array}$ \\
\hline & $F$ & Sig. & $t$ & $g l$ & (bilateral) & de medias & diferencia & Superior & Inferior \\
\hline $\begin{array}{l}\text { Se han } \\
\text { asumido } \\
\text { varianzas } \\
\text { iguales }\end{array}$ & 3.90 & .049 & -26.44 & 298 & .000 & -26.08 & .98 & -28.02 & -24.13 \\
\hline $\begin{array}{l}\text { No se han } \\
\text { asumido } \\
\text { varianzas } \\
\text { iguales }\end{array}$ & & & -26.44 & 283.8 & .000 & -26.08 & .98 & -28.02 & -24.13 \\
\hline
\end{tabular}




\section{Discusión y Conclusiones}

El objetivo de esta investigación fue determinar si existen diferencias en la percepción de equidad de género y en relación al equilibrio trabajo-familia en trabajadores pertenecientes a una empresa pública y una privada de Chile. Para esto, se plantearon tres hipótesis, las cuales serán descritas a continuación mediante la discusión de los resultados obtenidos.

En relación a la hipótesis 1 se propone que existen diferencias estadísticamente significativas en la percepción de equidad de género entre las empresas. En cuanto a las características de las mismas, se puede mencionar que éstas difieren en sus modos de operar.

Por un lado, pese al constante esfuerzo que presentan las organizaciones del Estado por alcanzar el bienestar de sus trabajadores, este se ve perjudicado por las distintas metas o propósitos que comparten los involucrados que toman las decisiones dentro de la organización, ya sea por las bruscas variaciones en el personal que compone la dirección producto de los cambios de gobierno, o por los objetivos esperados por los parlamentarios, los cuales pueden diferir del resto. A su vez, dentro de estas empresas existen grupos que buscan resolver sus propios intereses sin pensar en la opinión de los demás (Hernández \& Argimon, 2000). Por el contrario, las empresas privadas, cuentan con un trato directo por parte de los directivos con sus trabajadores, velando por la salud y bienestar de éstos con medidas de corto, mediano y largo plazo (Trujillo \& Vargas, 1996). Cabe señalar que estas organizaciones viven bajo un ritmo acelerado y dinámico, buscando las innovaciones para engrandecer sus beneficios (Merlano, 1983).

Por otro lado, de acuerdo con los resultados obtenidos en la aplicación del cuestionario SUSESO/ISTAS 21 el sector público mostró un mayor porcentaje de mujeres en sus organizaciones $(39.8 \%$ hombres y $60.2 \%$ mujeres) que el sector privado $(51.6 \%$ hombres y $48.4 \%$ mujeres), lo cual hace esperar que las percepciones difieran producto de la cantidad de trabajadoras que pertenecen a un sector y a otro (Superintendencia de Seguridad Social, 2017).

Los resultados obtenidos en este estudio confirman la existencia de diferencias estadísticamente significativas en la Percepción de Equidad de Género entre una empresa pública y privada $(p>.05)$, resultando que, los trabajadores pertenecientes a la empresa privada tienen una mayor percepción de equidad de género que los trabajadores pertenecientes a la empresa pública.

En los estudios realizados por Hofstede (1990) en 20 organizaciones públicas y privadas de Holanda y Dinamarca, se menciona que tales organizaciones difieren en cuanto a sus prácticas implementadas, las cuales tenían mucha relación con el sistema de control por parte de los directivos, la realización de la tarea y la jerarquización de la empresa. Por su parte, Gilbreath (2004) plantean que las políticas organizacionales tienen mejores resultados en los trabajadores, cuando éstas involucran una relación más personal entre dirección y subordinados, como las que se pueden observar en empresas del ámbito privado, creando así un grupo homogéneo dentro del cual, cada medida implementada, beneficiará al grupo completo. Las percepciones que los individuos tengan acerca de estas prácticas organizacionales y de las tareas llevadas a cabo dentro del ámbito laboral orientará a mejorar el clima dentro de la empresa (Goncalves, 2002).

La hipótesis 2 de este trabajo planteaba la existencia de diferencias estadísticamente significativas en relación al Equilibrio Trabajo-Familia entre una empresa pública y otra privada. La implementación de prácticas organizacionales que fomenten la conciliación laboral y familiar está ligada al trabajo que realizan las empresas, específicamente el área de recursos humanos, con el fin de proteger a sus empleados, otorgarles bienestar y satisfacción y evitar ausentismo o renuncias (Cappelli, 2000). Se espera que las empresas que integran el ámbito público y privado presenten diferencias debido a la gestión que éstas realizan, las cuales difieren una de la otra en su accionar. 
Los resultados expuestos en este estudio confirman la existencia de diferencias estadísticamente significativas en relación al Equilibrio Trabajo-Familia entre una empresa pública y otra privada $(p>.05)$, resultando que, los trabajadores pertenecientes a la empresa privada reportan un mayor equilibrio trabajo-familia que los trabajadores pertenecientes a la empresa pública.

Según la literatura revisada por Idrovo (2006) sobre estudios realizados en empresas privadas, se observa que las políticas organizacionales que integran estas instituciones van ligadas a la producción y trabajo eficiente por parte de los empleados, es decir implementan prácticas de conciliación trabajo-familia para retener a sus trabajadores más eficientes y aumentar la productividad. Además, éstas se relacionan con la satisfacción laboral del trabajador, disminución de su estrés y compromiso para y con la empresa. Por su parte Allen (2001) menciona que la percepción de apoyo que tienen los trabajadores por parte de la empresa tiene una relación positiva con la realización de las tareas que éstos tienen en sus dos ámbitos más importantes, el trabajo y la familia.

Por último, la hipótesis 3 planteaba que existe correlación positiva entre las variables Equidad de Género y Equilibrio Trabajo-Familia aplicadas a la muestra total. Según lo expuesto anteriormente, se puede señalar que darle solución al conflicto generado por intentar conciliar el trabajo y la familia, beneficiaría a las mujeres que pertenecen a la empresa, como también la implementación de prácticas como: trabajo a tiempo parcial y reducir la doble jornada, asumiendo responsabilidades compartidas, que se ligarían al principio de igualdad de género y al equilibrio trabajo-familia (Papí, 2005).

Los resultados observados en este estudio confirman la existencia de una correlación fuerte y positiva entre las variables Equidad de Género y Equilibrio Trabajo-Familia $(r=.796, p<$ .05) aplicadas a la muestra total.

La literatura revisada muestra que los cambios sociales y políticos que han ido ocurriendo, en cuanto a la redistribución de los roles del hombre y la mujer, han generado conflictos entre el trabajo y la familia. La necesidad de aumentar el ingreso al hogar ha ido modificando las dinámicas familiares, tales como el ingreso de la mujer al trabajo remunerado. El acceso al trabajo por parte de los dos padres genera problemas para conciliar el trabajo y la familia, no solo para la mujer, sino también para el hombre quien ha tenido que implicarse mayormente en las labores del hogar (Idrovo, 2006).

En cuanto a las limitaciones que manifiesta esta investigación, se puede señalar que se utilizó una muestra que no representa la totalidad de empresas, tanto públicas como privadas, del país, por lo cual los resultados no se pueden generalizar. Al mismo tiempo, no se podrían determinar diferencias entre estas empresas, incluyendo solamente los cuestionarios aplicados. A su vez, las instituciones comparadas pertenecen a distintos rubros, lo que podría interferir en los resultados obtenidos.

Pese a que las prácticas organizacionales están creadas para fomentar la motivación del trabajador, procurar su bienestar y reforzar su compromiso con la empresa, existe poca evidencia empírica en cuanto a cómo se llevan a cabo dentro de las organizaciones y si son factibles en estos ámbitos, generando así una limitación a la incorporación y creación de políticas en temas de equilibrio trabajo-familia y equidad de género.

Para esto se propone enfatizar en un diseño que incorpore un análisis más completo, abarcando técnicas cualitativas y cuantitativas, que incorpore la opinión de los entrevistados, entendiendo que, debido a los fuertes cambios psicosociales ocurridos a nivel mundial, las proyecciones de vida, tanto personal como profesional, son distintas entre un trabajador y otro, las cuales pueden interferir en los niveles de percepción de equidad de género o equilibrio trabajo-familia. Al mismo tiempo integrar otras variables como corresponsabilidad familiar beneficiará los resultados que se puedan extraer de tal diseño planteado, ya que asumir las tareas que conlleva un hogar entre el hombre y la mujer de manera equitativa, mejorará el equilibrio en 
los ámbitos de trabajo-familia (Dirección del Trabajo, 2015). Es necesario evaluar las prácticas orientadas a conciliar el trabajo y la familia dentro de las organizaciones, ya que éstas pueden estar dando solución al conflicto generado entre estos contextos mejorando la percepción de apoyo que tienen los trabajadores acerca de su empresa, es por esto que se sugiere integrar las variables cultura y apoyo organizacional para reforzar la eficacia en la que se llevan a cabo estas medidas. Por último, es importante evaluar el tipo de cargo que tienen las mujeres de las empresas estudiadas y las políticas que las empresas hayan implementado a la hora de aplicar el estudio, puesto que, según talleres de evaluación ciudadana aplicados por la ley, la percepción de inequidad de género se presenta mayormente en mujeres $(63 \%)$ que en hombres $(52 \%)$ viéndose reflejada en las organizaciones con cargos directivos o gerenciales, lo que se traduce en que la desigualdad de remuneración salarial perjudicaría más a las mujeres del ámbito profesional (Dirección del Trabajo, 2015), por lo cual las percepciones difieren según la jerarquía o estructura que la organización tenga.

\section{Referencias}

Abarca, N. y Errázuriz, M. (2007), Propuestas para la Conciliación Trabajo y Familia, en Camino al Bicentenario. Propuestas para Chile, Pontifica Universidad Católica. Santiago de Chile.

Abramo, L. \& Valenzuela, M. (2006). Inserción laboral y brechas de equidad de género en América Latina. En L. Abramo (Ed.), Trabajo decente y equidad de género en América Latina (pp. 9-62). Santiago, Chile: Organización Internacional del Trabajo.

Allen, T. (2001). Family-Supportive Work Environments: The role of organizational perceptions. Journal of Vocational Behavior, 58, 414-435. Doi: 10.1006 / jvbe.2000.1774

Anderson, S. E., Coffey, B. S., \& Byerly, R. T. (2002). Formal Organizational Initiatives and Informal Workplace Practices: Links to Work-Family Conflict and Job-Related Outcomes. Journal of Management, 28(6), 787-810. Doi: 10.1177/014920630202800605

Banco Mundial (2012). Informe Sobre el Desarrollo Mundial: Igualdad de Género y Desarrollo, Panorama General. Recuperado de: https://siteresources.worldbank.org/INTWDR2012/Resources/77781051299699968583/7786210-1315936231894/Overview-Spanish.pdf

Besarez, F., Jiménez, A. \& Riquelme, E. (2014). Apoyo organizacional hacia la familia, corresponsabilidad y satisfacción laboral según tipo de políticas organizacionales de equilibrio trabajo-familia. Trabajo y Sociedad, 23, 525-535.

Boles, J., Johnston, M., \& Hair, J. (1997). Role stress, work-family conflict and emotional exhaustion: Interrelationships and effects on some work-related consequences. Journal of Personnel Selling \& Sales Management, 17(3), 51-52. Doi: 10.1080 / 08853134.1997.10754079

Caballer, A., Peiró, J. M., \& Sora, B. (2011). Consecuencias de la inseguridad laboral. El papel modulador del apoyo organizacional desde una perspectiva multinivel. Psicothema, 23(3), 394-400.

Cappelli, P. (2000). A market driven approach to retaining talent. Harvard Business Review, 78(1), 103-111.

Comunidad Mujer. (2016). Iniciativa paridad de género en Chile. Documento técnico. Recuperado de: http://www.comunidadmujer.cl/wpcontent/uploads/2016/12/2016 _12_07-Documento-Ejecutivo-t\%C3\%A9cnico_VF.pdf

Comunidad Mujer. (2017). Para un Chile sostenible: 10 propuestas de género. Documento técnico. Recuperado de: http://www.comunidadmujer.cl/wpcontent/uploads/2017/09 Propuestas_Digital_PL-1.pdf

Dirección del Trabajo (2015). La Desigualdad Salarial entre hombres y mujeres. Cuadernos de 
investigación 55. Recuperado de: https://www.dt.gob.cl/portal/1629/articles105461_recurso_1.pdf

Durán, G. \& Kremerman, M. (2018). Los Verdaderos Sueldos de Chile: Panorama Actual del Valor de la Fuerza del Trabajo Usando la ESI 2017. Estudios de la Fundación Sol. Recuperado de: http://www.fundacionsol.cl/wp-content/uploads/2018/08/LosVerdaderos-Salarios-NESI-2017-1.pdf

Gilbreath, B. (2004). Creating healthy workplaces: The supervisor's role. In C. L. Cooper

\& I. T. Robertson (Eds.), International review of industrial and organizational psychology (pp. 93-118). Chichester, England: Wiley

Gómez, S. \& Martí, C. (2004). La incorporación de la mujer al mercado laboral: Implicaciones personales, familiares y profesionales, y medidas estructurales de conciliación trabajofamilia. Documentos de Investigación Núm. 557. Barcelona: IESE Business School, Universidad de Navarra.: 49.

Gómez, V. \& Jiménez, A. (2015). Corresponsabilidad familiar y el equilibrio trabajo-familia: Medios para mejorar la equidad de género. Polis, 14(40), 377-396.

Goncalves, A. (2002) Dimensiones del clima organizacional. Recuperado de: http://www.geocities.ws/janethqr/liderazgo/130.html

Greenglass, E. (1985). Psychological implications of sex bias in the workplace. Academic Psychology Bulletin, 7, 227-240.

Guerrero, J. (2003). Los roles no laborales y el estrés en el trabajo. Revista Colombiana de Psicología, 12, 73-84.

Heilman, M. E. (2001). Description and prescription: How gender stereotypes prevent women's ascent up the organizational ladder. Journal of Social Issues, 57. 657-673. Doi: $10.1111 / 0022-4537.00234$

Hernández, P., Argimón, I. \& González-Páramo, J. M. (2000). ¿Afecta la titularidad pública la eficiencia empresarial? evidencia empírica con un panel de datos del sector manufacturero español. España: Imprenta del Banco de España.

Hirata, H. \& Zariffian, P. (2007). El concepto de trabajo. Revista de Trabajo, 3(4), 33-36.

Idrovo, S. (2006). Las políticas de conciliación trabajo-familia en las empresas colombianas. Estudios gerenciales, 22(100), 49-70.

Instituto Nacional de Estadísticas (2015). Encuesta Suplementaria de Ingresos. Recuperado de: https://www.ine.cl/estadisticas/sociales/ingresos-y-gastos/encuesta-suplementaria-deingresos

Instituto Nacional de Estadísticas (2016). Enfoque estadístico: Género y empleo. Recuperado de: https://www.ine.cl/docs/defaultsource/laborales/ene/publicaciones /g\%C3\%A9nero-y-empleo---enfoque estad\%C3\%ADstico-mayo-2016.pdf?sfvrsn=4

Instituto Nacional de Estadísticas (2018). Compendio estadístico 2018. Recuperado de: https://www.ine.cl/docs/defaultsource/publicaciones/2018/bookcompendio2018.pdf?sfvrs $\mathrm{n}=\mathrm{fae} 156 \mathrm{~d} 2 \_5$

Jiménez, A., \& Moyano, E. (2008). Factores laborales de equilibrio entre trabajo y familia: medios para mejorar la calidad de vida. Revista Universum, 23(1), 116-133.

Lambert, E., Hogan, N. \& Barton, S. (2001). The Impact of Job Satisfaction on Turnover Intent: A Test of a Structural Measurement Model Using a National Sample of Workers. The Social Science Journal, 38, 233-250.

Lorenzini, P., Ceroni, G., De Urresti, A., Gutiérrez, H., Gutiérrez, R., Monckeberg, N., Robles, A. \& Sepúlveda, A. (2013). Evaluación de la ley 20.348: Resguarda el derecho a la igualdad de remuneraciones. Departamento de Evaluación de la Ley. Recuperado de: http://www.evaluaciondelaley.cl/foro_ciudadano/site/artic/ 20130325/asocfile/20130325153119/informe_ley_nro_20348.pdf

Marín, M., Infante, E. \& Rivero, M. (2002). Presiones internas del ámbito laboral y/o familiar 
como antecedente del conflicto trabajo-familia. Revista de Psicología Social, 17(1), 103112.

Maruani, M. (1993). La cualificación, una construcción social sexuada. Economía y Sociología del Trabajo, 21-22, 41-50.

Merlano, A. (1983). Motivación y productividad. Revista administración de personal. ACRIP, 3 , 31.

Moreno, B., Sanz, A., Rodríguez, A. \& Geurts, S. (2009). Propiedades psicométricas de la versión española del Cuestionario de Interacción Trabajo-Familia (SWING). Psicothema, 21, 331-337.

Organización para la Cooperación y Desarrollo Económicos [OCDE (2010)] Estudio Económico de Chile. Recuperado de https://www.oecd.org/centrodemexico/44493040.pdf

Papí, N. (2005). La conciliación de la vida laboral y familiar como proyecto de calidad de vida desde la igualdad. Revista Española de Sociología, 5, 91-107.

Riquelme, E., Rojas, A., \& Jiménez, A. (2012). Perspectivas analíticas sobre la dinámica social. Equilibrio trabajo, familia, apoyo familiar, autoeficacia parental y funcionamiento familiar percibidos por funcionarios públicos de Chile. Trabajo y sociedad, 18, 203-215.

Rubalcava, R. (2001). Evolución del ingreso monetario de los hogares en el período 1977-1994. En: J. Gómez de León. \& C. Rabell (Eds.). La población de México. Tendencias y perspectivas sociodemográficas hacia el siglo XXI (pp. 694-724). México: Fondo de Cultura Económica.

Servicio Nacional de la Mujer [SERAM (2003)]. Análisis de los costos y beneficios de implementar medidas de conciliación de vida laboral y familiar en la empresa. Departamento de Estudios y Estadísticas de SERNAM, 84. Recuperado de: https://estudios.sernam.cl/documentos/?eODYxNjMwAn\%C3\%A1lisis_de_los_costos_y _beneficios_de_implementar_medidas_de_concliliaci\%C3\%B3n_de_la_vida_laboral_y_ familiar_de_las_empresas.

Servicio Nacional de la Mujer [SERAM (2017)]. Caracterización de Acciones de Buenas Prácticas Laborales con Equidad de Género (BPLEG), desarrolladas en organizaciones públicas y privadas del país. Recuperado de: http://www.biblioteca.digital.gob.cl/bitstream/handle/123456789/3618/SERNAMEG\%20 2017-

Estudio\%20Caracterizacion\%20de\%20acciones\%20de\%20BPL\%20con\%20equidad\%20 de $\% 20$ genero.pdf?sequence $=1 \&$ isAllowed $=y$

Stevens, I. \& Van Lamoen, I. (2001). Manual on Gender Mainstreaming at Universtities. 'Equal Opportunities at Universities. Towards a Gender Mainstreaming Approach'. Garant: Leuven Apeldoorn.

Superintendencia de Seguridad Social. (2017). Riesgo psicosocial en Chile. Resultados de la aplicación del Cuestionario SUSESO/ISTAS21 (2016). Panorama Mensual Seguridad y Salud en el Trabajo, 3(12). Recuperado de: https://www.suseso.cl/607/articles480616_archivo_01.pdf

Trujillo, M. \& Vargas, D. (1996). Categorías motivacionales requeridas para mantener $e$ incrementar la productividad de trabajadores de empresas públicas y privadas de Santa Fe de Bogotá. Universidad Nacional de Colombia, Departamento de Psicología, Bogotá.

Contribución de los autores: a) Concepción y diseño del trabajo; b) Adquisición de datos; c) Análisis e interpretación de datos; d) Redacción del manuscrito; e) revisión crítica del manuscrito.

A.J.F. ha contribuido en a,b,c,d,e; A.H.R. en b,c,d

Editora científica responsable: Dra. Cecilia Cracco 